\title{
CRITICAL ASSOCIATED METRICS ON CONTACT MANIFOLDS
}

\author{
DAVID E. BLAIR \\ (Received 14 December 1982, revised 22 April 1983) \\ Communicated by K. Mackenzie
}

\begin{abstract}
Defining a function on the set of all Riemannian metrics associated to a contact form on a compact manifold by taking the integral of the Ricci curvature in the direction of the characteristic vector field, it is shown that on a compact regular contact manifold the only critical points of this function are the metrics for which the characteristic vector field generates a group of isometrics.
\end{abstract}

1980 Mathematics subject classification (Amer. Math. Soc.): 53 C 25.

\section{Introduction}

It is known that a certain amount of geometric information is carried by integrals of particular functions over a compact manifold, especially if such an integral is a critical value of a function defined by such integrals on a space of certain geometric objects over the manifold. For example, in the set of all Riemannian metrics of prescribed volume on a compact orientable $C^{\infty}$ manifold, a metric is an Einstein metric if and only if it is a critical point of the integral of the scalar curvature. It is our purpose here to begin the study of such integrals for integrands defined in terms of other structures and over certain subspaces of the space of metrics.

In [2] it was shown that a Riemannian metric $g$ associated to a contact form $\eta$ on a $(2 n+1)$-dimensional contact manifold is $K$-contact if and only if the Ricci curvature in the direction of the characteristic vector field $\xi$ is equal to $2 n$. In particular $R_{j i} \xi^{j} \xi^{i}=2 n-\operatorname{tr} h^{2}$ where $h$ is a symmetric operator described in [1]

(c) 1984 Australian Mathematical Society $0263-6115 / 84 \$ A 2.00+0.00$ 
and briefly below. It is clear then that on the space of all associated metrics over a compact contact manifold, the $K$-contact metrics are maxima for the integral

$$
I(g)=\int_{M} R_{j i} \xi^{j} \xi^{i} d V
$$

where $R_{j i}$ is computed with respect to $g$ and $d V=\left(1 / 2^{n} n !\right) \eta \wedge(d \eta)^{n}$. We will show that on a regular contact manifold the $K$-contact metrics are the only critical points of $I(g)$. Thus the condition of being $K$-contact in the contact case may be viewed as analogues to being Einstein in the Riemannian case. Specifically stated, our result is the following.

THEOREM. Let $M$ be a compact regular contact manifold with contact form $\eta$. Then the associated metric in the set of all metrics associated to $\eta$ is a critical point of the function I if and only if it is a K-contact metric.

While one might conjecture this for non-regular contact manifolds, the regular case is at least a natural context for this question as regular contact manifolds always carry a $K$-contact metric whereas a non-regular contact manifold may or may not carry one (see, for example, Chapter IV of [1]).

\section{Associated metrics}

By a contact manifold we mean a pair $(M, \eta)$ where $M$ is a $(2 n+1)$-dimensional $C^{\infty}$ manifold and $\eta$ is a global 1-form satisfying $\eta \wedge(d \eta)^{n} \neq 0$. Given the contact form $\eta$, there exists a unique vector field $\xi$ such that $\eta(\xi)=1$ and $d \eta(\xi, X)=0$ for all $X ; \xi$ is called the characteristic vector field of the contact structure $\eta$. To introduce associated metrics on $(M, \eta)$ we give the following two step process [6,1]. Let $k^{\prime}$ be any Riemannian metric on $M$ and define a metric $k$ by

$$
k(X, Y)=k^{\prime}(-X+\eta(X) \xi,-Y+\eta(Y) \xi)+\eta(X) \eta(Y) .
$$

$k$ is a Riemannian metric with respect to which $\eta$ is the covariant form of $\xi$. Let $X_{1}, \ldots, X_{2 n}, \xi$ be a local $k$-orthonormal basis and consider the $2 n \times 2 n$ matrix $\left(d \eta\left(X_{i}, X_{j}\right)\right)$; it is non-singular and hence can be written uniquely as a product $F G$ where $F$ is orthogonal and $G$ is positive definite and symmetric. $G$ then defines a Riemannian metric $g$ on $M$ by the matrix $\left(\begin{array}{ll}G & 0 \\ 0 & 1\end{array}\right)$, its components being given with respect to $X_{1}, \ldots, X_{2 n}, \xi$. Similarly $F$ defines a global tensor field $\phi$ of type $(1,1)$ by the matrix $\left(\begin{array}{l}F \\ 0 \\ 0\end{array}\right)$ and $\phi$ satisfies $\phi^{2}=-\mathrm{id}+\eta \otimes \xi$ and $\phi \xi=0 . g$ is called an associated metric of $\eta$ and $(\eta, g)$ or $(\phi, \xi, \eta, g)$ a contact metric structure. In particular $\phi$ and $g$ are so constructed that $d \eta(X, Y)=g(X, \phi Y)$. 
Given a contact metric structure $(\phi, \xi, \eta, g)$ we define a tensor field $h$ by $h=\frac{1}{2} \ell_{\xi} \phi$ where $\mathcal{L}$ denotes Lie differentiation. $h$ is a symmetric operator on $T M$ [1] and it is easy to see that the vector field $\xi$ is Killing with respect to $g$ if and only if $h=0$. A contact metric structure for which $\xi$ is Killing is called a $K$-contact structure. We also have the following general formulae, the first two of which are derived in [1] and the third in [3]; $\nabla$ denotes the Riemannian connection of $g$ and $R$ its curvature tensor.

$$
\begin{gathered}
\nabla_{X} \xi=-\phi X-\phi h X, \\
\frac{1}{2}\left(R_{\xi X} \xi-\phi R_{\xi \phi X} \xi\right)=h^{2} X+\phi^{2} X, \\
\left(\nabla_{\xi} h\right) X=\phi X-h^{2} \phi X-\phi R_{X \xi} \xi .
\end{gathered}
$$

Recall that the forms $\eta$ and $d \eta$ on a contact manifold are invariant under $\xi$, that is, $\mathscr{L}_{\xi} \eta=0$ and $\mathcal{L}_{\xi}(d \eta)=0$. Since the volume element for any associated metric is $d V=\left(1 / 2^{n} n !\right) \eta \wedge(d \eta)^{n}$ [7], we have $\mathcal{E}_{\xi} d V=0$; thus if $M$ is compact and $f$ any function on $M,(\xi f) d V=\mathscr{L}_{\xi}(f d V)=d(\iota(\xi) f d V)$ and hence

$$
\int_{M}(\xi f) d V=0
$$

Finally let $S^{2} M$ denote the bundle of symmetric tensors of type $(0,2)$ and $C^{\infty}\left(S^{2} M\right)$ the set of all smooth sections of $S^{2} M$. The set $\Re$ of Riemannian metrics is then the set of all $g \in C^{\infty}\left(S^{2} M\right)$ such that $g$ induces a positive definite inner product on each tangent space. Now the set $\mathcal{Q}$ of associated metrics can be shown to be a connected subset of $\mathfrak{R}$. Moreover $\mathcal{Q}$ is infinite dimensional as can be seen from the deformation used in Section 3 below. An associated metric $g$ is a critical point of the function $I$ on $Q$ given by (1.1), if $d I /\left.d t\right|_{t=0}=0$ for all smooth curves $g(t)$ in $\mathbb{Q}$ such that $g(0)=g$.

\section{Proof of the theorem}

As already noted, $K$-contact metrics are maxima of the function $I$, so our task is to prove that these are the only critical points of $I$.

Let $g(t)$ be a smooth curve in $\mathcal{Q}$ and define several tensors by

$$
\begin{gathered}
D_{j i}=\left.\frac{\partial g_{j i}}{\partial t}\right|_{t=0}, \\
D_{j i}{ }^{h}=\frac{1}{2}\left(\nabla_{j} D_{i}^{h}+\nabla_{i} D_{j}^{h}-\nabla^{h} D_{j i}\right), \\
D_{k j i}{ }^{h}=\nabla_{k} D_{j i}{ }^{h}-\nabla_{j} D_{k i}{ }^{h},
\end{gathered}
$$


where $\nabla$ denotes the Riemannian connection of $g(0)$. Then

$$
\left.\frac{\partial R_{k j i}{ }^{h}}{\partial t}\right|_{t=0}=D_{k j i}^{h}
$$

where $R_{k j i}{ }^{h}$ denotes the curvature tensor of $g(t)$ (see, for example, [5]). Since the metrics $g(t)$ are associated to a fixed contact form $\eta$,

$$
D_{j i} \xi^{j}=\frac{\partial g_{j i}}{\partial t} \xi^{j}=\frac{\partial \eta_{i}}{\partial t}=0
$$

and

$$
D_{j i} \phi_{k}^{j}=\frac{\partial g_{j i}}{\partial t} \phi_{k}^{j}=-g_{j i} \frac{\partial \phi_{k}^{j}}{\partial t}
$$

from which

$$
D_{l}^{i}=\phi_{l}^{k}, \frac{\partial \phi_{k}^{i}}{\partial t}=-\phi_{k}^{i} \frac{\partial \phi_{l}^{k}}{\partial t} .
$$

In particular we see that the tensor field $D$ of type $(1,1)$ and the tensor field $\phi$ corresponding to $g(0)$ anti-commute and hence that tr $D=0$.

Now $d I /\left.d t\right|_{t=0}=\int_{M} D_{k j i}{ }^{k} \xi^{j} \xi^{i} d V$ and we proceed to compute the integrand. First of all using equation (2.1)

$$
D_{j i}{ }^{k} \xi^{j}=\frac{1}{2}\left(\xi^{j} \nabla_{j} D_{i}^{k}+D_{j}^{k}\left(\phi_{i}^{j}+\phi_{m}^{j} h_{i}^{m}\right)-D_{j i}\left(\phi^{j k}+\phi_{m}^{j} h^{m k}\right)\right)
$$

giving $D_{j k}{ }^{k} \xi^{j}=0$ and also, since $h \xi=0, D_{j i}{ }^{k} \xi^{j} \xi^{i}=0$. Therefore

$$
\begin{aligned}
D_{k j i}{ }^{k} \xi^{j} \xi^{i}= & \left(\nabla_{k} D_{j i}{ }^{k}-\nabla_{j} D_{k i}{ }^{k}\right) \xi^{j} \xi^{i} \\
= & 2 D_{j i}{ }^{k}\left(\phi_{k}^{i}+\phi_{m}^{i} h^{m}{ }_{k}\right) \xi^{j} \\
= & \left(\xi^{j} \nabla_{j} D_{i}^{k}+D_{j}^{k}\left(\phi^{j}{ }_{i}+\phi^{j}{ }_{m} h^{m}{ }_{i}\right)-D_{j i}\left(\phi^{j k}+\phi^{j}{ }_{m} h^{m k}\right)\right) \\
& \times\left(\phi_{k}^{i}+\phi_{l}^{i} h_{k}^{l}\right) \\
= & \xi\left(D_{i}^{k}\left(\phi_{k}^{i}+\phi_{l}^{i} h_{k}^{l}\right)\right)-D_{i}{ }^{k} \phi_{l}^{i} \xi^{j} \nabla_{j} h_{k}^{\prime}+2 D_{i k} h^{i k}
\end{aligned}
$$

where the last step uses the fact that $\nabla_{\xi} \phi=0$ and $\phi h+h \phi=0$ (see, for example, [1]). In view of equation (2.4) we need only consider the last two terms in continuing the computation. Using equation (2.3), $D_{i}{ }^{k} \xi^{i}=0$ and $\operatorname{tr} D=0$, the integrand becomes

$$
\left(-h^{i}{ }_{l}^{\prime}{ }_{k}-R_{k r s}{ }^{i} \xi^{s} \xi^{s}+2 h^{i}{ }_{k}\right) D^{k}{ }_{i} .
$$

Thus if $g(0)$ is a critical point of $I, d I /\left.d t\right|_{t=0}=0$ and hence

$$
\int_{M}\left(-h_{l}^{i} h^{i k}-R_{r s}^{k} \xi^{i} \xi^{s}+2 h^{i k}\right) D_{i k} d V=0 .
$$


Suppose now that the above computation has been done with respect to a local $\phi$-basis defined on a neighborhood $U$ (that is, the basis consists of $\xi, X_{1}, \ldots, X_{2 n}$, orthonormal with respect to $g=g(0)$ and $\left.X_{2 i}=\phi X_{2 i-1}\right)$. If now $X$ is any unit vector field on $U$ orthogonal to $\xi, X$ is part of a $\phi$-basis and call it $X_{1}$ for simplicity in indexing. Consider the two-dimensional subspaces determined by $X_{1}$ and $X_{2}=\phi X_{1}$ and let $f$ be a $C^{\infty}$ function with compact support in $U$. Then define a curve $g(t)$ of metrics given by the following deformation in these two-dimensional subspaces over $U$ by the matrix

$$
\left(\begin{array}{rr}
1+t f+\frac{1}{2} t^{2} f^{2} & \frac{1}{2} t^{2} f^{2} \\
\frac{1}{2} t^{2} f^{2} & 1-t f+\frac{1}{2} t^{2} f^{2}
\end{array}\right) .
$$

It is easy to check that $g(t)$ is a curve in $\mathcal{Q}$ and clearly the only non-zero components of $D_{i j}$ are $D_{11}=-D_{22}=f$. Thus since $f$ is any $C^{\infty}$ function with support in $U$, equation (3.1) gives

$$
-h_{l}^{1} h^{\prime 1}-R_{r s}^{1}{ }_{r s} \xi^{r} \xi^{s}+2 h^{11}+h^{2} h^{12}+R_{r s}^{2}{ }^{2} \xi^{r} \xi^{s}-2 h^{22}=0
$$

or since $X$ was any unit vector field orthogonal to $\xi$

$$
\begin{aligned}
& -g\left(h^{2} X, X\right)-K(X, \xi)+2 g(h X, X) \\
& \quad+g\left(h^{2} \phi X, \phi X\right)+K(\phi X, \xi)-2 g(h \phi X, \phi X)=0
\end{aligned}
$$

where $K(X, \xi)$ is the sectional curvature of the plane section spanned by $X$ and $\xi$. Equation (2.2) and the fact that $\phi$ and $h$ anti-commute then give

$$
g\left(R_{X \xi} \xi, X\right)=g\left(X-h^{2} X+2 h X, X\right) .
$$

Thus

$$
R_{X \xi} \xi=-\phi^{2} X-h^{2} X+2 h X+T X
$$

where for the endormorphism $T, g(T X, X)=0$. On the other hand the identity $g\left(R_{X \xi} \xi, Y\right)=g\left(R_{Y \xi} \xi, X\right)$ shows that $T$ is symmetric and hence $T=0$. Therefore we have for any critical associated metric

$$
R_{X \xi} \xi=-\phi^{2} X-h^{2} X+2 h X
$$

for all $X$.

Next we draw some elementary conclusions from equation (3.2). Combining it with equation (2.3) we have

$$
\left(\nabla_{\xi} h\right) X=-2 \phi h X .
$$

If now $X$ is an eigenvector of $h$ with eigenvalue $\lambda$,

$$
0=g\left(\left(\nabla_{\xi} h\right) X, X\right)=g\left(\nabla_{\xi} \lambda X-h \nabla_{\xi} X, X\right)=(\xi \lambda) g(X, X)
$$


and therefore the eigenvalues of $h$ are constant along the integral curves of $\xi$ and so also their multiplicity. Similarly taking the inner product of equation (3.3) with $\phi X$,

$$
-2 \lambda g(X, X)=g\left(\lambda \nabla_{\xi} X-h \nabla_{\xi} X, \phi X\right)=2 \lambda g\left(\nabla_{\xi} X, \phi X\right)
$$

so for $\lambda \neq 0$ and $X$ a unit eigenvector

$$
g\left(\nabla_{\xi} X, \phi X\right)=-1 .
$$

Now suppose that $M$ is a regular contact manifold, then $M$ is a principal circle bundle with $\xi$ tangent to the fibres [4]. Locally $M$ is $U \times S^{1}$ where $U$ is a coordinate neighborhood on the base manifold. Recalling again that $\phi$ and $h$ anti-commute let $\xi, X_{2 i-1}, X_{2 i}=\phi X_{2 i-1}$ be an orthonormal eigenvector basis of $h$ at some point of $U \times S^{1}$. If we continue this basis along the fibre through this point, since the eigenvalues are constant along the fibre, there will be at worst a change in the orientation of some of the eigenspaces when we return to the starting point. So the basis may be continued smoothly around the fibre to within a possible reversal of direction upon return. Thus if $Y$ is a vector field along $\xi$ and we write $Y=\Sigma_{i}\left(\alpha_{2 i-1} X_{2 i-1}+\beta_{2 i} X_{2 i}\right)+\gamma \xi$ along any arc of the fibre, the coefficients $\alpha_{2 i-1}, \beta_{2 i}$ will be periodic functions of the parameter, though the period may be twice the length of the fibre.

If now the critical point $g$ is not a $K$-contact metric, some eigenvalue of $h$ is non-zero somewhere on $M$, say $\lambda_{2 i-1} \neq 0$ on some $U \times S^{1}$ as above. Again since $M$ is regular we may choose a vector field $Y$ on $U \times S^{1}$ such that $\alpha_{2 i-1} \not 0$ and $Y$ is horizontal and projectable, that is, $[\xi, Y]=0$. Writing $Y=\Sigma_{i}\left(\alpha_{2 i-1} X_{2 i-1}+\right.$ $\beta_{2 i} X_{2 i}$ ) along a fibre we have

$$
\begin{aligned}
& \begin{array}{l}
0[\xi, Y]=\nabla_{\xi} Y-\nabla_{Y} \xi \\
=\sum_{i}\left\{\left(\xi \alpha_{2 i-1}\right) X_{2 i-1}+\alpha_{2 i-1} \nabla_{\xi} X_{2 i-1}+\left(\xi \beta_{2 i}\right) X_{2 i}+\beta_{2 i} \nabla_{\xi} X_{2 i}\right. \\
\left.\quad \quad+\alpha_{2 i-1} X_{2 i}+\lambda_{2 i-1} \alpha_{2 i-1} X_{2 i}-\beta_{2 i} X_{2 i-1}+\lambda_{2 i-1} \beta_{2 i} X_{2 i-1}\right\}
\end{array}
\end{aligned}
$$

where $\nabla_{X_{2 i-1}} \xi=-\left(1+\lambda_{2 i-1}\right) X_{2 i}$ and $\nabla_{X_{2 i}} \xi=\left(1-\lambda_{2 i-1}\right) X_{2 i-1}$ follow from equation (2.1). Taking the inner product with $X_{2 i-1}$ and $X_{2 i}$ respectively and using equation (3.4) we have respectively

$$
\begin{aligned}
& \xi \alpha_{2 i-1}=-\lambda_{2 i-1} \beta_{2 i}, \\
& \xi \beta_{2 i}=-\lambda_{2 i-1} \alpha_{2 i-1} .
\end{aligned}
$$

Therefore $\xi \xi \alpha_{2 i-1}-\lambda_{2 i-1}^{2} \alpha_{2 i-1}=0$ contradicting the periodicity of $\alpha_{2 i-1}$. 


\section{References}

[1] D. E. Blair, Contact manifolds in Riemann geometry (Lecture Notes in Mathematics, 509, Springer, Berlin, 1976).

[2] D. E. Blair, 'Two remarks on contact metric structures,' Tôhoku Math. J. 29 (1977), 319-324.

[3] D. E. Blair and J. N. Patnaik, 'Contact manifolds with characteristic vector field annihilated by the curvature,' Bull. Inst. Math Acad. Sinica 9 (1981), 533-545.

[4] W. M. Boothby and H. C. Wang, 'On contact manifolds,' Ann. of Math. 68 (1958), 721-734.

[5] Y. Muto, 'On Einstein metrics,' J. Differential Geometry 9 (1974), 521-530.

[6] S. Sasaki, 'On differentiable manifolds with certain structures which are closely related to almost contact structure I,' Tôhoku Math. J. 14 (1962), 146-155.

[7] S. Sasaki, Almost contact manifolds (Lecture Notes, Mathematical Institute, Tôhoku University, Vol. 1, 1965).

\section{Department of Pure Mathematics}

The University of Liverpool

Liverpool, England

Author's current address:

Department of Mathematics

Michigan State University

East Lansing, Michigan

U.S.A. 